\title{
Atrial pacing for rhythm control of atrial fibrillation
}

\author{
I.C. van Gelder, A.C.P. Wiesfeld
}

A fter atrial fibrillation (AF) develops, the first step is to search for and treat underlying (heart) diseases. Thereafter, AF should be treated. This includes prevention of cardiovascular morbidity and mortality, especially vascular events, and reduction of symptoms. ${ }^{1}$ The latter may be obtained by two treatment strategies: rhythm-control and/or rate-control treatment. Recent randomised trials have shown that rate control is not inferior to rhythm control with regard to cardiovascular morbidity and mortality. ${ }^{2}$ In these studies, predominantly elderly patients with underlying heart diseases (especially hypertension) were included. Patients with (severely) symptomatic AF and advanced heart failure were excluded. Since then, rate-control treatment has been adopted more frequently, even as first-choice therapy, especially in the elderly.

Rhythm control, however, remains indicated in patients who are symptomatic with the arrhythmia. The Achilles' heel in the rhythm-control management of AF is that recurrences frequently occur. Nowadays, efficacy of catheter ablation of AF is increasing and this treatment strategy is gaining popularity rapidly, especially in younger patients with paroxysmal AF. Atrial pacing is another non-pharmacological option to prevent AF.

In a subset of patients paroxysmal AF develops in the setting of a sick sinus syndrome. These patients need atrial pacing. Studies have demonstrated that the use of atrial-based pacing compared with ventricular pacing significantly lowers the incidence of AF but does not improve survival nor heart failure or cardio-

\section{I.C. van Gelder}

Department of Cardiology, University Medical Center Groningen, University of Groningen, and the Interuniversity Cardiology Institute Netherlands, Utrecht, the Netherlands

A.C.P. Wiesfeld

Department of Cardiology, University Medical Center Groningen, University of Groningen, the Netherlands

Correspondence to: I.C. van Gelder,

Department of Cardiology, Thoraxcenter, University Medical

Center Groningen, University of Groningen, PO Box 30.001,

9700 RB Groningen, the Netherlands

E-mail: i.c.van.gelder@thorax.umcg.nl

vascular death. ${ }^{3}$ While atrial pacing is effective in preventing development of $\mathrm{AF}$ in patients with symptomatic bradycardia, its value as a treatment for paroxysmal AF in patients without conventional indications for pacing has not been proved. ${ }^{1}$ Novel pacemakers have several special algorithms implemented in order to prevent AF even more successfully, and are capable of measuring the AF burden. ${ }^{4}$ Prospective studies using these algorithms have yielded mixed results in patients with and without pacing indications. Some showed a reduction in AF burden, but the absolute difference was small. There was no gain in terms of quality of life, as well as mean number of AF episodes, hospitalisations, or mean duration of AF detected by the pacemaker's automatic mode switching algorithm. ${ }^{5,6}$ Others have failed to show any benefit of atrial pacing in preventing $\mathrm{AF},{ }^{7,8}$

Why should atrial-based pacing be effective in preventing AF? It may prevent bradycardia-induced dispersion of repolarisation and it may suppress triggers such as atrial premature beats. In addition, by maintaining AV synchrony, retrograde ventriculoatrial conduction is prevented that can cause valvular regurgitation and stretch-induced changes in atrial electrophysiology. Furthermore, as septal pacing seems most effective, shortening of total atrial conduction time and reduction of dispersion of atrial refractoriness and conduction velocity, i.e. modification of the substrate, may be essential.

In this issue of the Netherlands Heart Journal De Voogt and Van Hemel question whether reliable detection and diagnosis of atrial tachyarrhythmias is actually achievable with the current pacemaker algorithms and leads. ${ }^{9}$ They nicely elucidate the presence of significant technical pitfalls that weaken the accuracy of the diagnosis of atrial tachyarrhythmias by these pacemakers at present. These limitations include 1 ) inappropriate sensing of $\mathrm{AF}$ due to variable and low voltage signals during $\mathrm{AF}$, which may cause inappropriate detection of start and/or perpetuation of $\mathrm{AF}$ and other atrial arrhythmias; 2 ) far-field $\mathrm{R}$ wave sensing which may be prevented by adaptation of other pacemaker parameters which, in turn, may not only obscure far-field $\mathrm{R}$ wave sensing but also every second 
beat of an atrial tachycardia; and 3 ) insufficient memory capacity of the pacemaker to store all atrial arrhythmia episodes. They state that 'major improvement in pacemaker diagnostics is mandatory before pace intervention in atrial arrhythmias can be put to the test in largescale trials.'

We agree and also recognise these shortcomings. To prevent AF by pacing, first of all, AF should be accurately diagnosed. Only then can pace intervention adequately be judged for its efficacy. Alternatively, diagnostic tools may be used to detect aymptomatic AF, e.g. possibly of importance for the assessment of the risk of stroke. ${ }^{10}$ Though technical pitfalls are certainly present, as the authors state themselves, the total duration of AF burden measured with a pacemaker seems highly reliable. ${ }^{11,12}$ Certainly, improvement in technical features will further enhance the accurate use of these diagnostic tools. But is it worthwhile to put every effort into improving this technology in order to use it for rhythm control for AF, also in patients without a pacing indication? Will there be a role for primary therapy for prevention of recurrent AF? The efficacy of atrial-based pacing in patients with a sick sinus syndrome probably just relates to prevention of bradycardias. It remains to be proven if additional pacing features have supplementary efficacy and whether these will ever play a role in patients without conventional pacing indications.

\section{References}

l Fuster V, Ryden LE, Cannom DS, Crijns HJ, Curtis AB, Ellenbogen KA, et al. ACC/AHA/ESC 2006 Guidelines for the Management of Patients with Atrial Fibrillation: a report of the American College of Cardiology/American Heart Association Task Force on Practice Guidelines and the European Society of Cardiology Committee for Practice Guidelines (Writing Committee to Revise the 2001 Guidelines for the Management of Patients With Atrial Fibrillation): developed in collaboration with the European Heart Rhythm Association and the Heart Rhythm Society. Europace 2006;8:651-745.
2 Van Gelder IC, Hagens VE, Bosker HA, Kingma JH, Kamp O, Kingma T, et al. A comparison of rate control and rhythm control in patients with recurrent persistent atrial fibrillation. $N$ Engl J Med 2002;347:1834-40.

3 Healey JS, Toff WD, Lamas GA, Andersen HR, Thorpe KE, Ellenbogen KA, et al. Cardiovascular outcomes with atrial-based pacing compared with ventricular pacing: meta-analysis of randomized trials, using individual patient data. Circulation 2006;114: $11-7$.

4 Israel CW, Hugl B, Unterberg C, Lawo T, Kennis I, Hettrick D, et al. Pace-termination and pacing for prevention of atrial tachyarrhythmias: results from a multicenter study with an implantable device for atrial therapy. J Cardiovasc Electrophysiol 2001;12: 1121-8.

5 Carlson MD, Ip J, Messenger J, Beau S, Kalbfleisch S, Gervais P, Cameron DA, et al. A new pacemaker algorithm for the treatment of atrial fibrillation - Results of the Atrial Dynamic Overdrive Pacing Trial (ADOPT). J Am Coll Cardiol 200;42:627-33.

6 Hemels MEW, Ruiter JH, Molhoek GP, Veeger NJGM, Wiesfeld ACP, Ranchor AV, et al. Right atrial preventive and antitachycardia pacing for prevention of paroxysmal atrial fibrillation in patients without bradycardia: a randomized study. Europace 2008;10: 306-13.

7 Padeletti L, Purerfellner H, Adler SW, Waller TJ, Harvey M, Horvitz L, et al. Combined efficacy of atrial septal lead placement and atrial pacing algorithms for prevention of paroxysmal atrial tachyarrhythmia. J Cardiovasc Electrophysiol 2003;14:1189-95.

8 Lee MA, Weachter R, Pollak S, Kremers MS, Naik AM, Silverman $\mathrm{R}$, et al. The effect of atrial pacing therapies on atrial tachyarrhythmia burden and frequency: results of a randomized trial in patients with bradycardia and atrial tachyarrhythmias. J Am Coll Cardiol 2003;41:1926-32.

9 De Voogt WG, Van Hemel NM. Diagnostic tools for atrial tachyarrhythmias in implantable pacemakers. A review of technical options and pitfalls. Neth Heart J 2008;16:201-10.

10 Hohnloser SH, Capucci A, Fain E, Gold MR, Van Gelder IC, Healey J, et al. Asymptomatic atrial fibrillation and stroke evaluation in pacemaker patients and the atrial fibrillation reduction atrial pacing trial (ASSERT). Am Heart J 2006;152:442-7.

11 Nowak B, McMeekin J, Knops M, Wille B, Schroder E, Moro C et al. Validation of dual-chamber pacemaker diagnostic data using dual-channel stored electrocardiograms. Pacing Clin Electrophysiol 2005;28:620-9.

12 De Voogt WG, Van Hemel NM, Van de Bos AA, Koistinen J, Fast $\mathrm{JH}$. Verification of pacemaker automatic mode switching for the detection of atrial fibrillation and atrial tachycardia with Holter recording. Europace 2006;8:950-61. 